\title{
Distributive Tactile sensing using Fibre Bragg grating sensors
}

\author{
Barbara M. Cowie ${ }^{\mathrm{a}}$, David J. Webb ${ }^{\mathrm{a}}$, Betty Tam ${ }^{\mathrm{b}}$, Paul Slack ${ }^{\mathrm{b}}$, Peter N. Brett ${ }^{\mathrm{b}}$ \\ ${ }^{a}$ Photonics Research Group, Aston University, Birmingham UK. B4 7ET \\ ${ }^{\mathrm{b}}$ Clinical Biomedical Engineering Research Group, Aston University, Birmingham, UK. B4 7ET
}

\begin{abstract}
Two distributive tactile sensing systems are presented, based on fibre Bragg grating sensors. The first is a onedimensional metal strip with an array of 4 sensors, which is capable of detecting the magnitude and position of a contacting load. This system is compared experimentally with a similar system using resistive strain gauges. The second is a two-dimensional steel plate with 9 sensors which is able to distinguish the position and shape of a contacting load. This system is compared with a similar system using 16 infrared displacement sensors. Each system uses neural networks to process the sensor data to give information concerning the type of contact.
\end{abstract}

Keywords: Bragg, Distributive, Sensor, Neural, Optical.

\section{INTRODUCTION}

\subsection{Tactile sensing}

Artificial tactile sensing is a highly sought after goal. While the rapidly falling cost of machine vision systems based on video imaging has meant that automatic identification of objects in many applications can now be achieved visually, there are still applications where artificial tactile sensing is greatly desirable. Examples of these include harsh environments where video equipment is impracticable, the assessment of soft deformable objects or surface textures, and minimal access surgery where tactile feedback would be invaluable to the surgeon in assessing the condition of organs and tissues.

Human tactile sensing is achieved by means of at least four different types of receptor cells ${ }^{1}$, and is used to feel, grasp and manipulate objects, and to assess attributes such as shape, size, texture, temperature, hardness, discontinuities such as holes or edges, and movement, including vibration.

Attempts to produce artificial tactile sensors usually rely on the measurement of pressure or force on a sensing element. A variety of different types of sensors have been used, including resistive strain gauges ${ }^{2}$, piezoelectric film ${ }^{3}$, infrared displacement sensors ${ }^{4}$, capacitive sensors ${ }^{5}$, sensors detecting conductance ${ }^{6}$, magnetic, magnetoelectric, and ultrasonic sensors ${ }^{7}$. In this paper we describe the use of fibre Bragg grating (FBG) strain sensors.

Frequently large numbers of sensing elements are built into an array and the outputs of those sensors are processed, often in conjunction with a mathematical model, to give an assessment of the contacting object. One example comprises a 64 element array with a sensitive area of $3 \mathrm{~cm}^{2} 3 \mathrm{~cm}^{8}$. While this approach is necessary for the small scale identification of fine form objects, scaling up these arrays to cover more extensive areas would result in very large numbers of individual sensors, a complex construction, many connections (or the need to multiplex), and a high computational load. Distributive tactile sensing by contrast, uses a small number of sensors. These sensors monitor a homogenous flexible substrate whose bending behaviour is reliable and repeatable, and a neural network interprets the sensor output data, having been previously trained to discriminate between different kinds of contact ${ }^{9}$. The substrate which the sensors monitor is an integral part of the sensing system. The sensors could be embedded within the substrate, bonded onto its surface (as in this case) or fixed beneath the substrate to monitor its deflection ${ }^{4}$. The use of a neural network to interpret the sensor output data means that whereas the standard tactile sensing approach uses sensors whose outputs are independent of each other and ideally linear, distributive tactile sensing uses sensors whose outputs exhibit a high degree of cross coupling and are not necessarily linear. The neural network recognizes patterns in the data from the group of sensors as a whole rather than considering the sensor outputs individually.

Biophotonics and New Therapy Frontiers, edited by Romualda Grzymala, Olivier Haeberlé Proc. of SPIE Vol. 6191, 61910Z, (2006) · 1605-7422/06/\$15 · doi: 10.1117/12.662597 


\subsection{Fibre Bragg grating sensors}

Fibre Bragg grating sensors ${ }^{10}$ can be used to sense strain, temperature and a number of other measurands. In this paper they are used exclusively as strain sensors. Temperature effects were minimised by the use of an air-conditioned laboratory, thermal isolation of the load from the sensing surface, and by taking unloaded measurements immediately before or after loaded measurements so that temperature compensation could be achieved.

\subsection{Neural Networks}

The type of neural network used was a Multilayer Perceptron with a single hidden layer. This consists of a collection of nodes, each of which calculates a weighted sum of its inputs, and applies an activation function to the result (figure 1). The nodes are arranged in layers: an input layer, one or more hidden layers, and an output layer. The nodes of one layer receive their inputs from all nodes of the previous layer, and their outputs are in turn connected to every node in the following layer (figure 2).

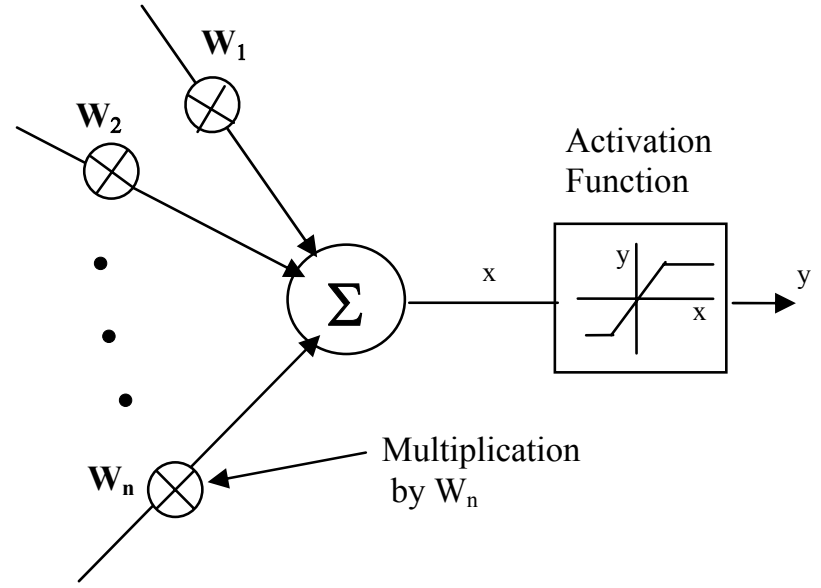

Figure 1: The structure of a Neural Network node

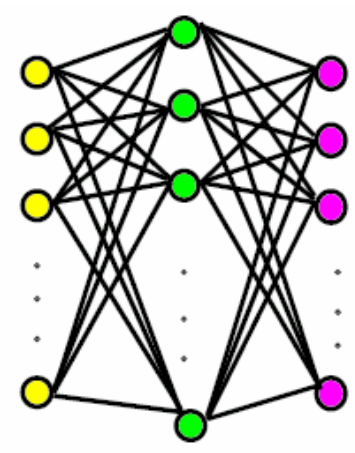

Inputs $\begin{gathered}\text { Hidden Outputs } \\ \text { Layer }\end{gathered}$

Figure 2: A Multilayer Perceptron with a single hidden layer

There is no reliable analytical method for predicting the best architecture (i.e. number of hidden layers and nodes) to use for a given problem, except that normally there should be more hidden nodes than input nodes (or at least as many), and that one hidden layer is sufficient to model any continuous function. The optimal architecture for any given application has to be arrived at by presenting the data to a range of networks in turn and finding out which produces the lowest residual training error. The software used in this case was NetLab, a collection of Neural Network tools used with MATLab and written by the Neural Computing Group at Aston University ${ }^{11}$. Training a neural network to recognise patterns in data of high dimensionality usually requires large amounts of training data, ideally including all possible combinations of the variables involved. Using a neural network involves the following processes:

1. Scaling the data

2. Choosing the activation function

3. Training the network

4. Choosing the optimum architecture

The input variables used in a neural network should be scaled so that they have similar magnitudes. The rescaled variables should have zero mean and unit variance. This helps to prevent the network training to become stuck in a local optimum, rather than finding the best values for the weights ${ }^{12}$. When initialising a neural network it is necessary to choose the activation function, which determines the type of output produced by the neural network. Some of the options available are Linear, Logistic and Softmax models. In a linear regression output neural network, the output will be in continuous numerical form, a Logistic Model allows the output to be in one of two classes, a softmax model puts output data into two or more classes. Table 1 shows 1-N encoding for a three-class model. 


\begin{tabular}{|l|lll|}
\hline Class 1 & 1 & 0 & 0 \\
\hline Class 2 & 0 & 1 & 0 \\
\hline Class 3 & 0 & 0 & 1 \\
\hline
\end{tabular}

Table 1 1-N Encoding for a three-class softmax model

A neural network is trained by presenting to it a set of training data (sets of sensor output values together with their respective load positions) and a second similar set of validation data. During each training cycle the internal constants or 'weights' used by the neural network are adjusted by the training software so that the error between the load position predicted by the neural network for the validation data inputs and the actual load position is minimised (figure 3 ).

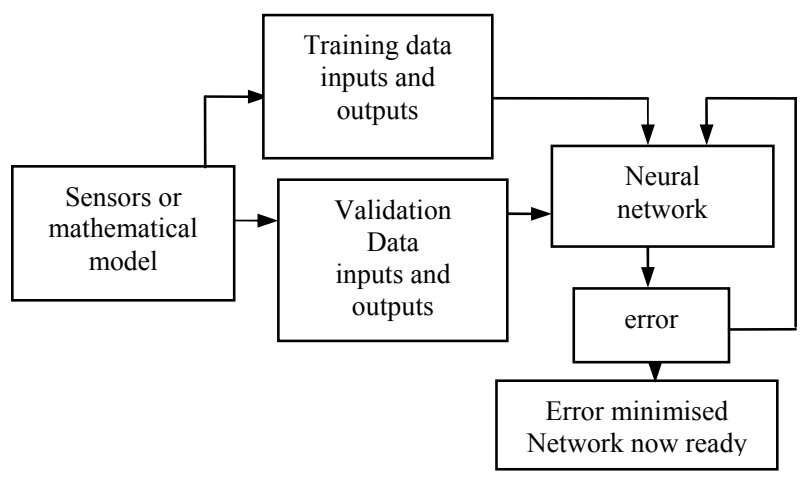

Figure 3: Schematic diagram of Neural Network training

A training epoch comprises at least as many cycles as there are weights in the network. The training error for the network typically minimises and then gradually increases (figure 4).

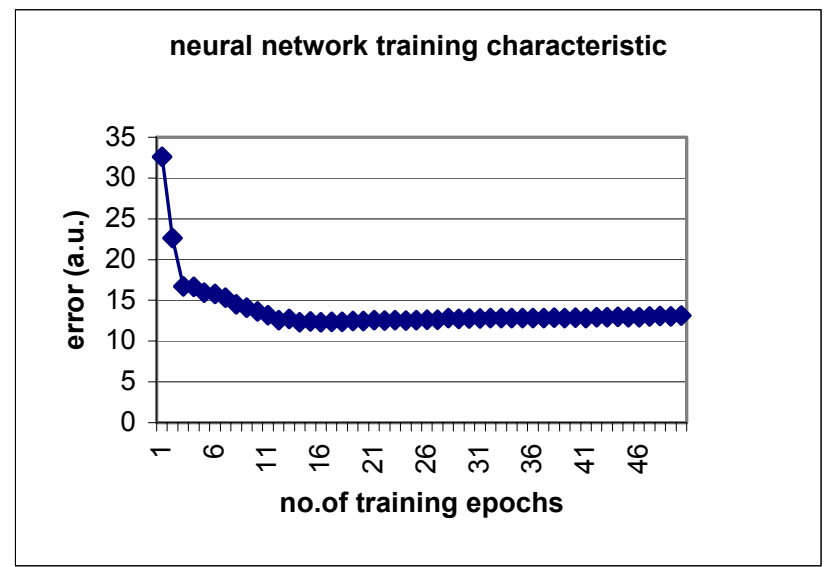

Figure 4: Training a Neural Network

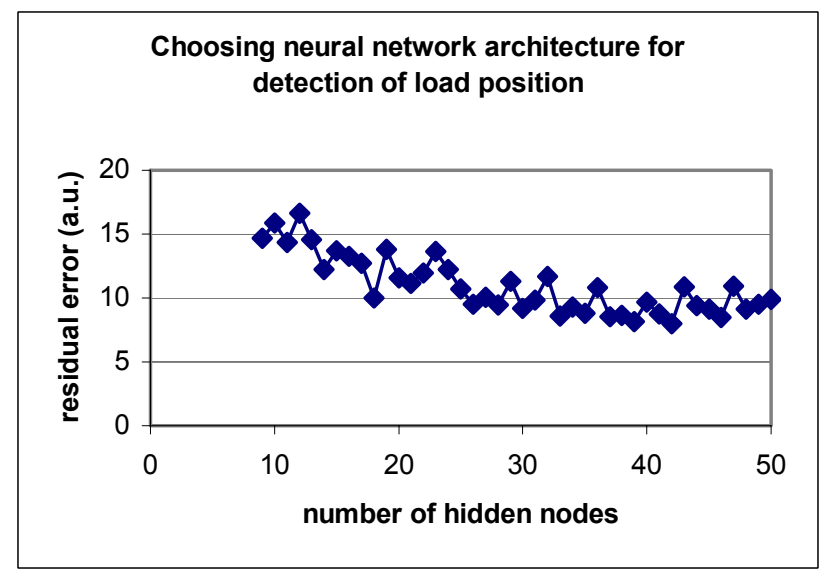

Figure 5: Optimising the number of hidden nodes

The architecture is optimised by assessing the performance of a large number of possible alternatives The residual training error minimises at a certain number of hidden nodes (figure 5). This enables the best neural network to be chosen for the task.

\section{ONE-DIMENSIONAL TACTILE SENSING SYSTEM}

First we discuss the application of this technology to a one-dimensional system, which was developed as a step towards the ultimate aim of providing an endoscope with tactile sensitivity ${ }^{9}$, and compare the performance of fibre Bragg grating strain sensors with that of conventional resistive strain gauges. 
A steel strip (figure 6) was prepared and four fibre Bragg grating sensors were fixed to it using epoxy adhesive. The locations of these sensors had previously been optimised using a genetic algorithm which selected the sensor positions which would give the maximum values of the first four principle components of the sensor outputs ${ }^{13}$. The sensors were multiplexed, having different reflective wavelengths so that they were contained within one fibre with a single connection (figure 7). A second identical steel strip had four resistive strain gauges fitted to it in the same positions and having 8 connecting wires (figure 8 ).

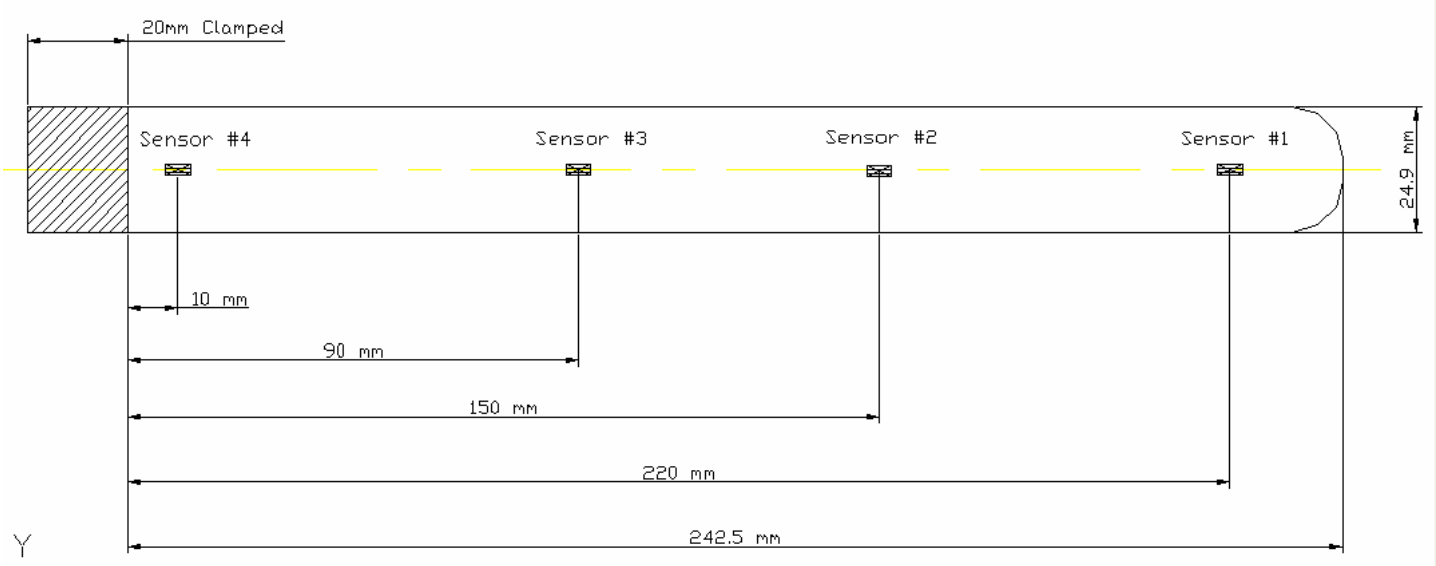

Figure 6: Sensor layout on 1-D tactile sensing strip.

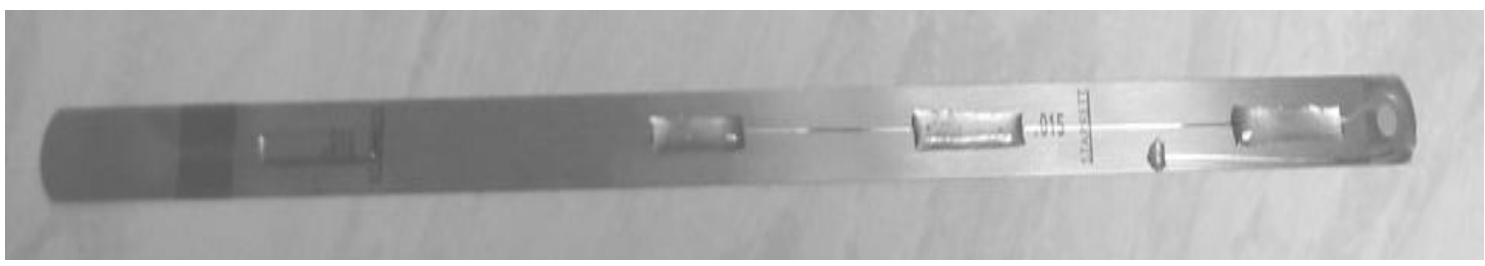

Figure 7: Steel strip with FBG sensors.

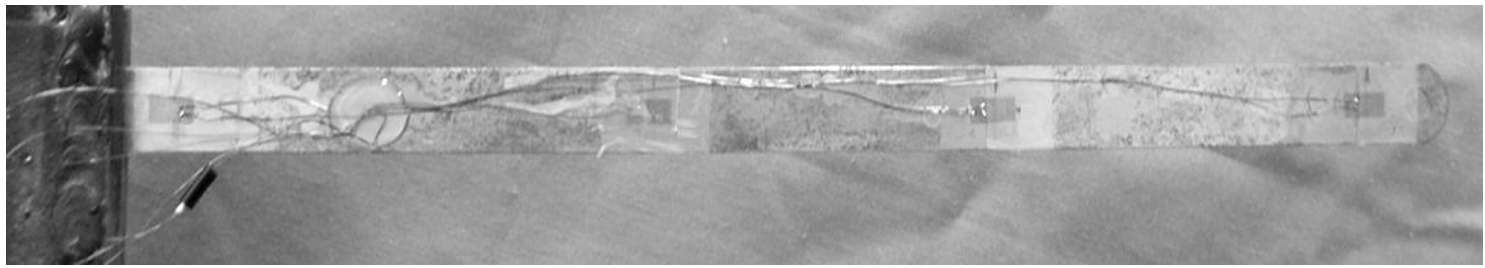

Figure 8: Steel strip with strain gauges

Interrogation of FBGs (figure 9) was achieved by launching broadband light into the fibre. The spectrum of the reflected light (figure 10) was measured by an optical spectrum analyser (OSA) and this information was processed by a PC running Labview, which calculated the wavelengths of the nine spectral peaks using an algorithm. The algorithm used in this case calculated the average wavelength at half the maximum amplitude of each spectral peak.

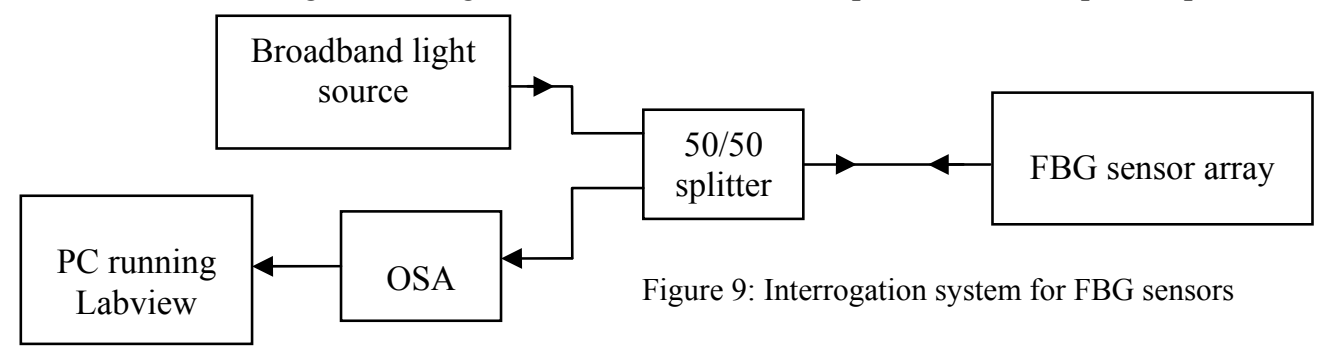




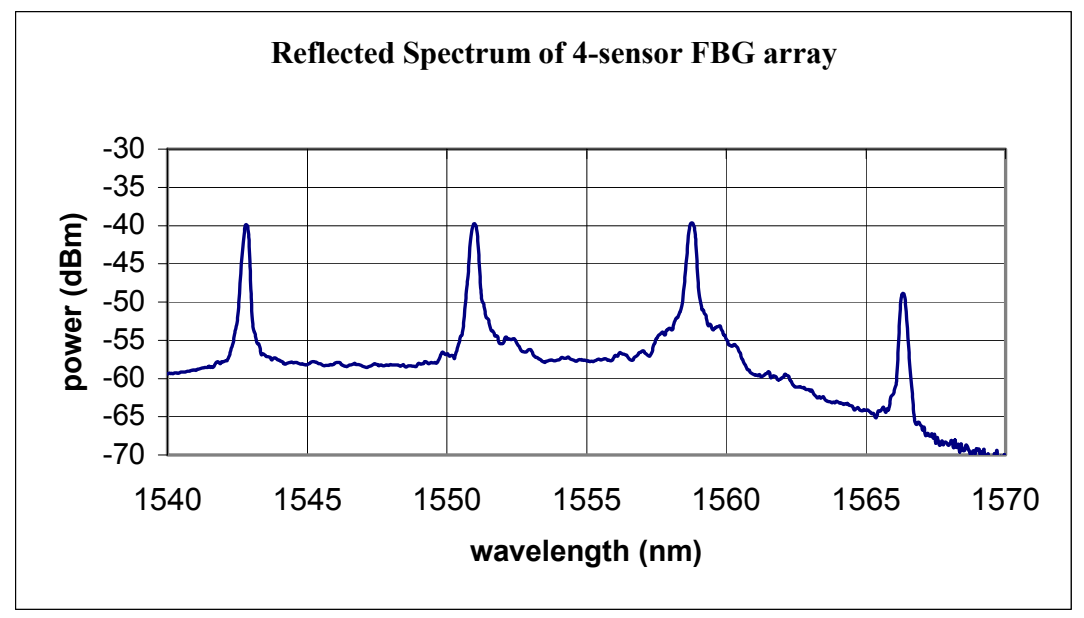

Figure 10: Reflected spectrum of 4-sensor FBG array

The resistive strain gauges were interrogated using a D.C. bridge circuit and electronic amplifiers. The steel strip was clamped at one end and a load was placed at a known distance along the strip. The sensor outputs were recorded. This was repeated for positions at $1 \mathrm{~cm}$ intervals along the strip and for a single, double and triple load.

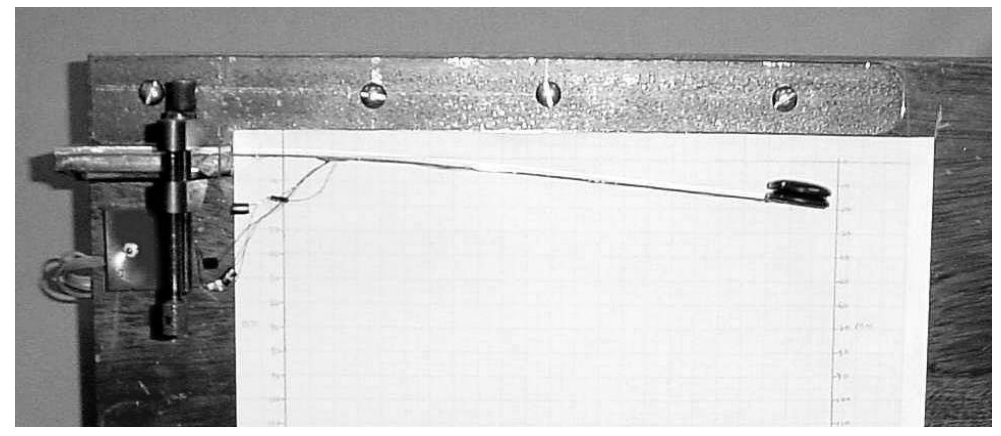

Figure 11: Clamped steel strip with double load on one end

Multiple sets of sensor data were recorded for each load position and each load size. The sensor output data were randomly allocated into three sets. One set was used to train the neural network, one for validation and the third set was used as test data to assess how well the trained neural network could distinguish the position and magnitude of the load. This was repeated for the second steel strip so that the performance of the systems using the two different sensor types could be compared.

The performance of the two sensor types was assessed in 3 ways.

1. Ability to detect the presence or absence of any load at any position.

2. Accuracy of distinguishing between the three load magnitudes (any load position).

3. Accuracy of detection of load position (any magnitude of load).

The optimal neural network architecture chosen for each parameter is shown below (table 2).

\begin{tabular}{|l|l|c|}
\hline Type Of Neural Network & Type of Output & Optimal No. Hidden Nodes \\
\hline Linear (4 Inputs) & Position (FBG) & 15 \\
\hline Logistic (4 Inputs) & Contact Present (FBG) & 45 \\
\hline Softmax (4 Inputs) & Load Magnitude (FBG) & 10 \\
\hline Linear (4 Inputs) & Position (Strain) & 5 \\
\hline Logistic (4 Inputs) & Contact Present (Strain) & 60 \\
\hline Softmax (4 Inputs) & Load Magnitude (Strain) & 15 \\
\hline
\end{tabular}

Table 2 - Neural Network architectures chosen for one-dimensional system 


\subsection{Results}
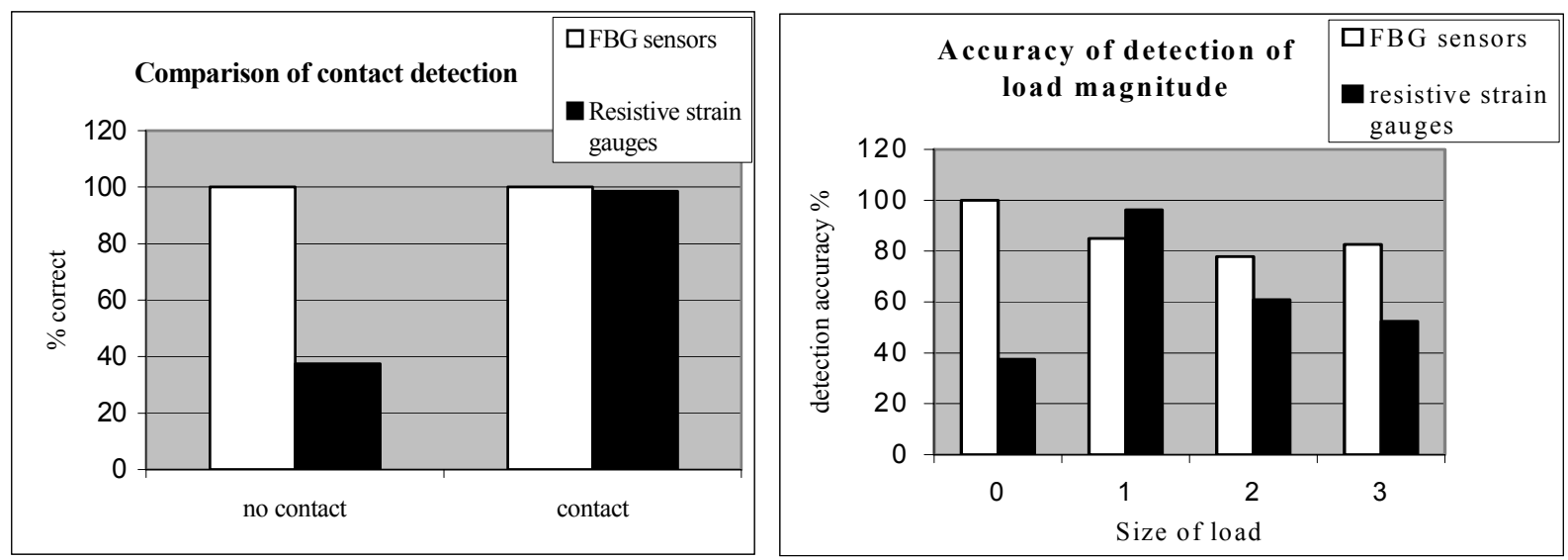

Figure 12: Comparisons of contact and load magnitude detection $1^{15}$

Figure 12 shows that the system using data from FBG sensors performed better than the system using data from resistive strain gauges in both contact/non contact detection and in discriminating between 3 out of 4 of the load sizes. In particular the resistive strain gauge system did not perform well in unloaded conditions.

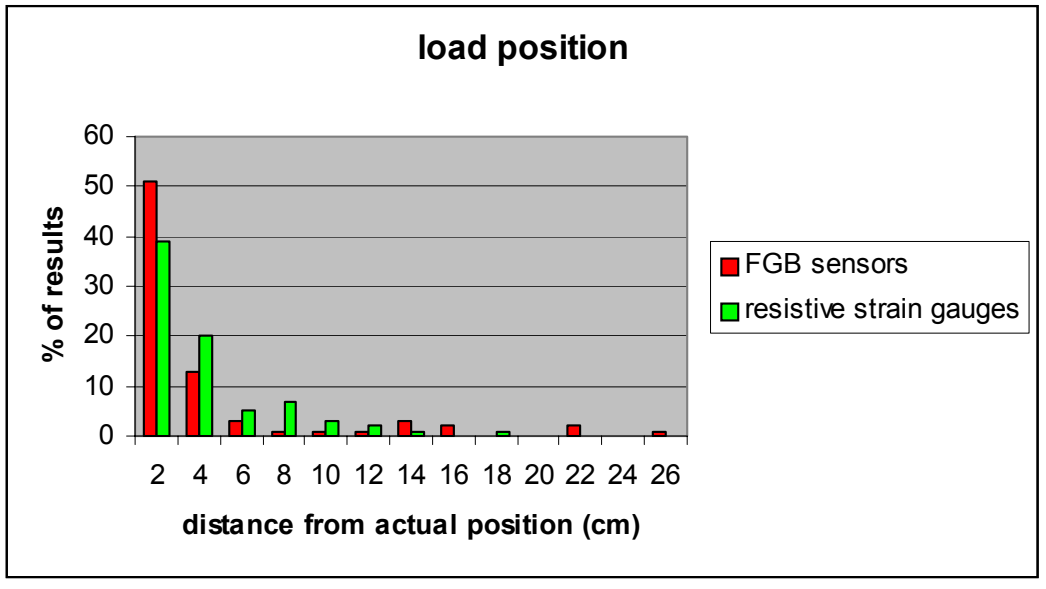

Figure 13: Comparison of load position detection

Figure 13 shows that the system using FBG sensors was able to detect the position of the load to within $2 \mathrm{~cm}$ of its actual position more than $50 \%$ of the time. The system using resistive strain gauges achieved less than $40 \%$ in the same test.

\section{TWO-DIMENSIONAL TACTILE SENSING SURFACE}

Having established the suitability of fibre Bragg grating sensors for use in this sensing regime, we moved on to study a 2 dimensional 'smart surface', comprising a simply supported stainless steel plate, which has a sensitive area of dimensions $160 \mathrm{~mm}$ x 280mm (figure 14).

Nine fibre Bragg grating sensors were mounted onto the underside of a stainless steel plate using epoxy adhesive. 


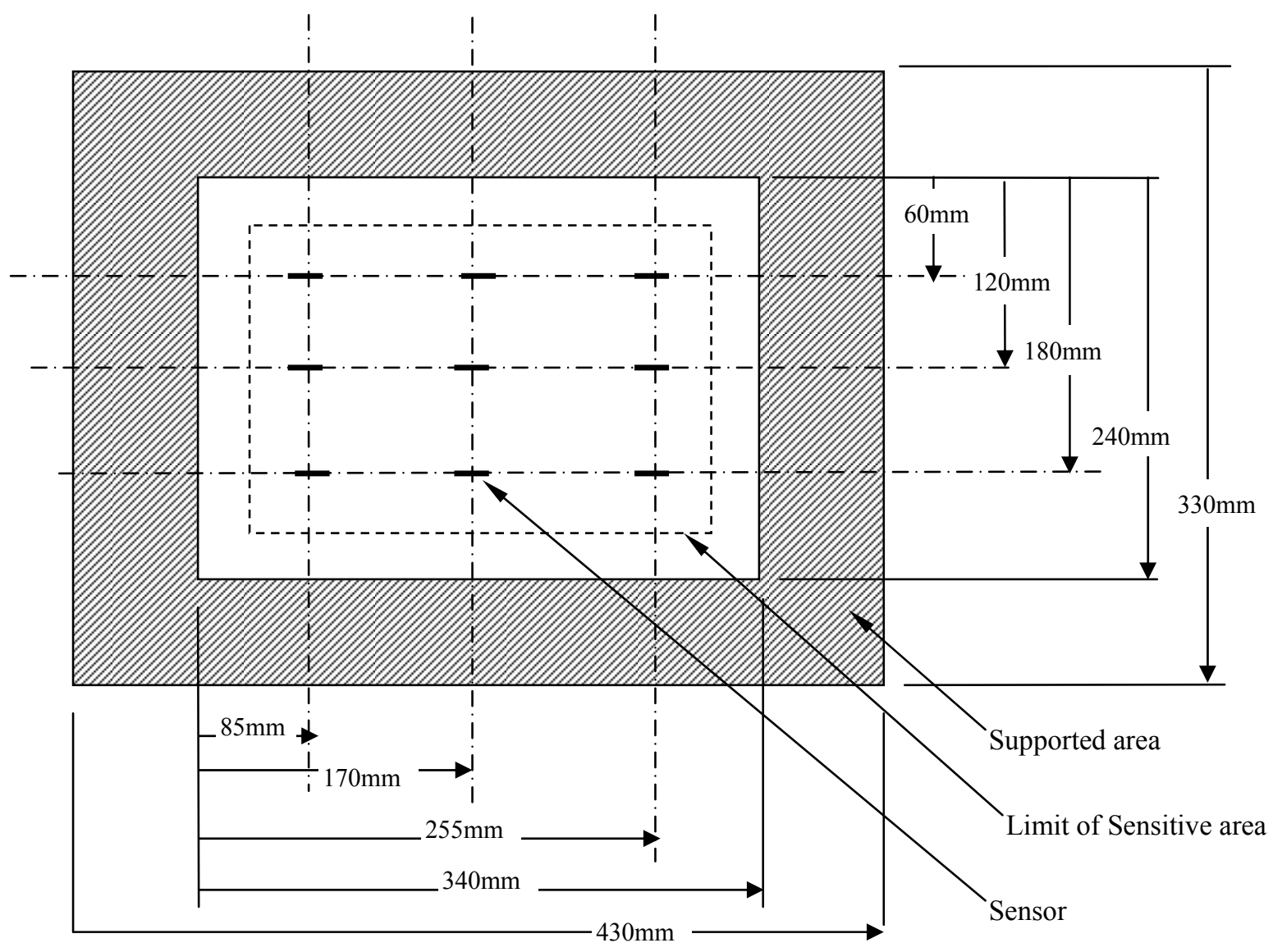

Figure 14 Layout of sensors on 2 Dimensional tactile surface

The plate was simply supported by a framework (figure 15) which allowed the plate to deflect downwards when a load was applied.

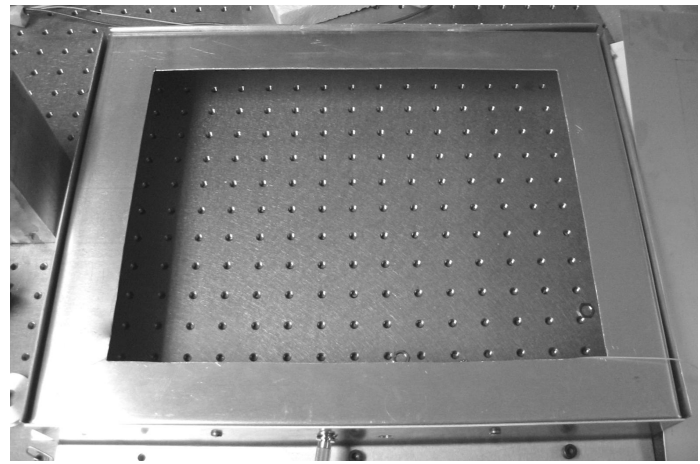

Figure 15 Supporting structure for tactile surface

The smart surface was first trained to recognise the position of a load placed upon it. Many systems using neural networks use theoretically derived data with added Gaussian 'noise' to increase the spread of the training data. Here we used experimentally collected data to train, validate and test the system. Those schemes which do use experimentally collected data for training frequently collect one batch of data, allocating it into subsets for training, validation and testing. Here the test data was collected independently of the training and validation data to more closely reflect the conditions of a practical system. A load was applied to the plate at all possible positions on a $20 \mathrm{~mm}$ grid, avoiding the area within $40 \mathrm{~mm}$ of the supporting structure to eliminate edge effects. At each load position the peak sensor output 
wavelengths were recorded. Three sets of data were recorded at each load position, for training, validation and testing respectively.

Neural networks with numbers of hidden nodes between 9 (the number of inputs to the networks) and 50 were trained. The error was found to minimize for each network within 150 training epochs.

\subsection{Results}

The surface was able to detect the load position with an rms error of approximately $11 \mathrm{~mm}(2.7 \%$ of full scale). The largest errors were generally found to be around the edges of the surface (figure 16), possibly because fewer of the sensors were significantly active when the load was close to the edge of the plate By comparison a similar arrangement using 16 infrared displacement sensors 14 had an average error of $4.5 \%$.

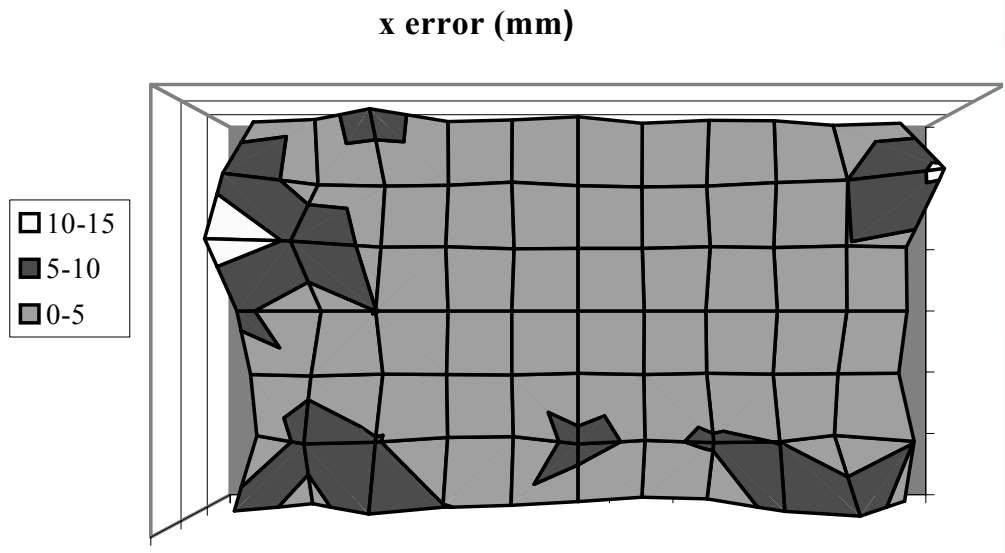

Figure 16: Map showing error $(\mathrm{mm})$ in load position detection

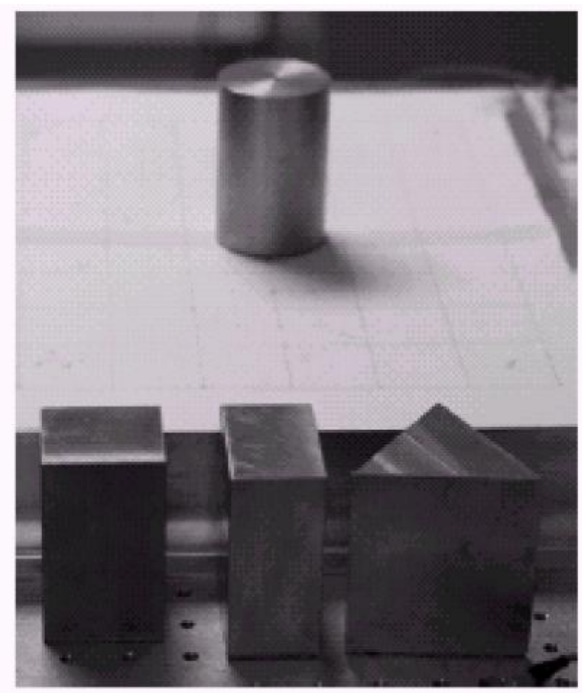

Figure 17: Shapes on sensing surface

Next the system was trained to recognise the shape of a load, using four shapes of equal area and weight (figure 17) Initially the load was placed at a set position, and the shapes were distinguished successfully $100 \%$ of the time. Then the range of positions on the surface at which the load was placed was increased until the entire sensitive area of the plate was used. The shape of a load at any position, with the position known, was detected with an accuracy of 91\% (figure 18). The shape of a load at any position, with the position unknown, was detected with $89 \%$ accuracy (figure 19).

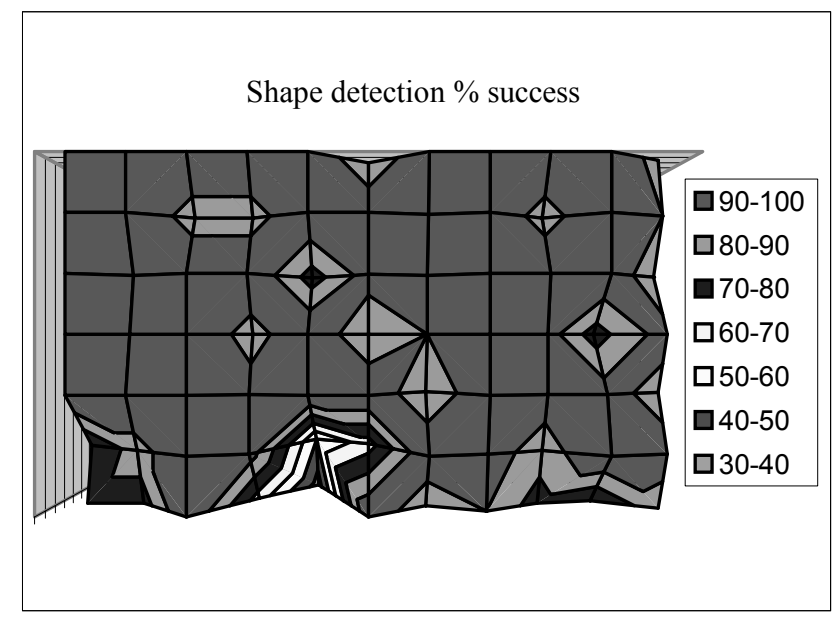

Figure 18: Map showing shape detection success rate

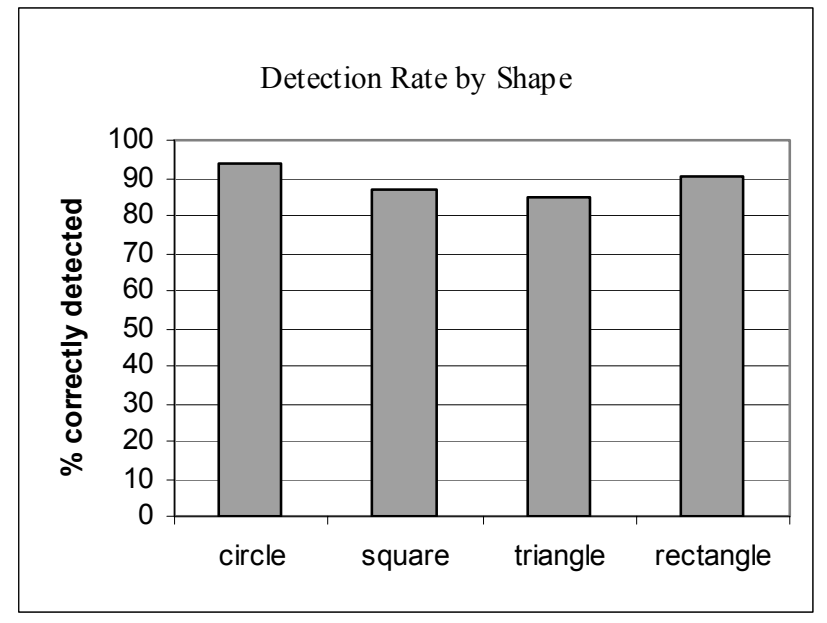

Figure 19: Shape detection rate by shape 


\subsection{Simultaneous shape and position detection}

There are two possible approaches to simultaneously detecting 2 different types of parameters using neural networks (in this case a continuous measurement of position and a shape classification task). It is not possible to use a single network to perform both a linear regression task and a classification task. One is to use a parallel arrangement, where one network processes the data and predicts the load position while another predicts the load shape (Fig. 20). The second approach is to use a cascaded arrangement where one network determines the load position and then that information is fed along with the sensor information into a second neural network which determines the shape (Fig. 21).

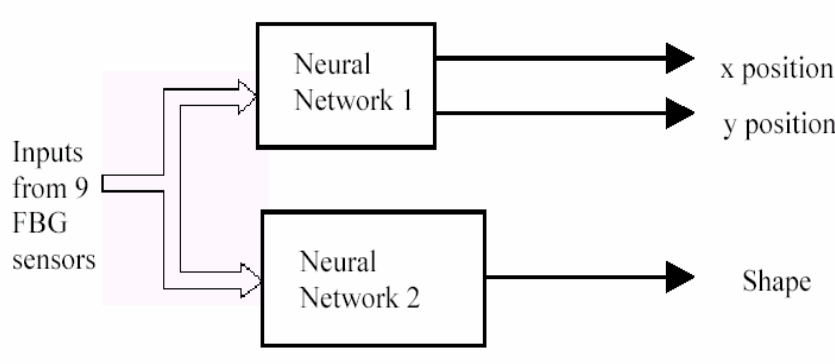

Figure 20: Parallel Networks

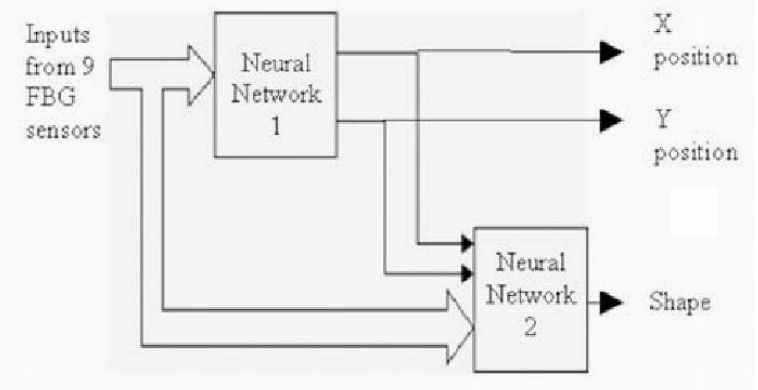

Figure 21: Cascaded Networks

The result above (89\% shape detection accuracy) is effectively from the parallel arrangement. The cascaded arrangement gave a shape detection accuracy of only $85 \%$. This shows that the neural network is capable of detecting the shape of a load without the additional information regarding the load position. It is likely that the decrease in accuracy in the second case is due to the errors in detecting the load position causing a decrease in load shape detection accuracy.

\section{CONCLUSIONS}

Fibre Bragg gratings have been successfully used in one and two-dimensional distributive tactile sensing systems, offering performance comparable to or better than conventional sensors. Distributive Tactile sensing systems use fewer sensors than conventional sensing regimes and use a fast mathematical algorithm, suitable for operation in real time applications. Fibre Bragg grating sensors offer advantages over other sensor types, they need fewer connections and are immune to electromagnetic noise, they can be embedded within materials without loss of material strength and can operate at high temperatures. They have particular advantages for use in endoscopic applications, being small and light, and having the possibility of being used in conjunction with MRI (Magnetic Resonance Image) scanning, opening up opportunity for performing an operation with continual scanning. Possible applications for the 2-dimensional sensing surface include human balance and gait monitoring (for which existing systems use large numbers of sensors or complex imaging systems), industrial pick and place applications and security systems. The detection of shape is not a continuous function so may benefit from a neural network with more than 2 layers of weights.

\section{ACKNOWLEDGEMENTS}

Barbara Cowie is supported by the UK Engineering and Physical Sciences Research Council

\section{REFERENCES}

1. B V Jayawant, “Tactile sensing in robotics”, Journal of Physics. E: Scientific. Instruments, v.22, no.9, pp. 684-692,. 1989.

2. R.E. Ellis, S.R. Ganeshan and S.J. Lederman, "A tactile sensor based on thin-plate deformation" Robotica, v. 12 pt.4, pp. 343-51, 1994.

3. J. Dargahi, M. Parameswaran and S. Payandeh, "A micromachined Piezoelectric Tactile Sensor for an endoscopic grasper - Theory, Fabrication and Experiments", Journal of Microelectromechanical Systems, v. 9, n. 3, pp. 329-35, 2000 
4. R.W.S. Stone, P. Tongpadungrod and Brett, P.N., ” A deforming sensing surface for evaluation of contact conditions", Engineering in Medicine and Biology Society, Proceedings of the 20 th Annual International Conference of the IEEE, v. 4, pp.1735-1737, 1998.

5. F. Castelli, "Integrated tactile-thermal robot sensor with capacitive tactile array" IEEE Transactions on Industry Applications, v. 38, no. 1, pp.85-90, 2002

6. E.G.M. Holweg and W. Jongkind, "Object recognition using a tactile matrix sensor", Proceedings EURISCON '94. European Robotics and Intelligent Systems Conference, v.3, pp 1379-83, 1994.

7. H.R. Nicholls and M.H. Lee, "A survey of robot tactile sensing technology", International Journal of Robotics Research, v 8, n 3, pp. 3-30, 1989.

8. K.B. Lim and Y.S. Chong, "Low cost tactile gripper using silicone rubber sensor array", Robotica, v 6, pt.1, pp. 2330, 1988.

9. I. Petra, D.J. Holding, K.J. Blow, B. Tam, X. Ma and P.N. Brett, "The design of a flexible digit towards wireless tactile sense feedback", Proceedings of 8th Int. Conf. on Control, Automation, Robotics and Vision (ICARCV), v. 1, pt.1, pp. 468-73, 2004.

10. Bennion, J.A.R. Williams, L. Zhang, K. Sugden and N.J Doran,"UV-written in-fibre Bragg gratings," Optical and Quantum Electronics, vol. 28, no. 2, pp. 93-135, 1996.

11. I. Nabney and C. Bishop. NetLab Toolbox Release 3.2 (2001), http://www.ncrg.aston.ac.uk/netlab/index.php (accessed $4^{\text {th }}$ Jan 2006).

12. C. M. Bishop, Neural Networks for Pattern Recognition, Oxford, Clarendon Press, 1995, p.29.

13. P. Tongpadungrod, T.D.L. Rhys,. and P.N. Brett, "An approach to optimise the critical sensor locations in onedimensional novel distributive tactile surface to maximise performance", Sensors and Actuator A: Physics, v.105, pp.47-54, 2003.

14. P. Tongpadungrod, “Characteristics of Distributive Tacile sensing systems”, PhD Thesis, University of Bristol, UK., 2001.

15. B. Tam and P. Slack, "Using Fibre Bragg Gratings as Strain Sensors with Neural Networks: A Comparison with using conventional Strain Sensors", Research report, Clinical Biomedical Engineering Research Group, Aston University, Birmingham, UK. 